\title{
Herramientas de análisis para la gestión de intercambiadores urbanos de transporte
}

\author{
Sara Hernández del Olmo ${ }^{1}$ \\ Dr. Ing. Caminos, Canales y Puertos, Consultora de Transportes en ARUP, España
}

\section{RESUMEN}

Hoy en día, dado el contexto y los patrones de movilidad cada vez más complejos de las ciudades, se evidencia una clara necesidad de mejorar el atractivo del transporte público. Muchos usuarios se ven obligados a combinar varios modos o servicios de transporte. Esto significa que los intercambiadores urbanos se han convertido en una experiencia cotidiana para los usuarios y, por lo tanto, desempeñan un papel clave para reducir los inconvenientes de la transferencia. El principal objetivo de este trabajo es presentar las herramientas de análisis desarrolladas junto con los principales resultados obtenidos en los tres intercambiadores de transporte europeos seleccionados como casos de estudio. Los resultados de esta investigación ponen de manifiesto la naturaleza ambivalente de los intercambiadores. Los factores funcionales contribuyen a hacer más fácil la transferencia y reducir el tiempo percibido de espera, mientras que los factores psicológicos hacen que la estancia sea más cómoda para los usuarios.

\section{INTRODUCCIÓN}

Actualmente, más de la mitad de la población mundial reside en áreas urbanas, y según un informe emitido por Naciones Unidas esta tendencia se espera que siga creciendo (Heilig, 2012). Este claro y continuo crecimiento deriva en modelos de ciudad complejos con un gran número de residentes, áreas de negocio, modos de transporte, servicios y utilidades conectados entre sí (Neirotti et al., 2014).

La tendencia hacia modelos de ciudad cada vez más dispersa, junto a este crecimiento sostenido de la población urbana ha supuesto un cambio en los patrones de movilidad. En consecuencia, las distancias y los tiempos de viaje se han incrementado drásticamente (Banister, 2011), obligando a muchos usuarios del transporte público a combinar varios modos y servicios de transporte para completar sus viajes diarios. El mayor reto de las ciudades es por tanto conseguir una mejora e incremento de la movilidad y al mismo tiempo reducir problemas como la congestión, los accidentes y la contaminación (COM, 2006).

Un principio básico para lograr este propósito es reducir los inconvenientes y molestias asociados a la transferencia o ruptura del viaje. En este sentido, es crucial establecer qué factores optimizan y determinan la competitividad de una terminal multimodal. A nivel urbano, son los intercambiadores de transporte los elementos vitales para fomentar la redistribución modal (Lopez-Lambas and Monzon, 2010), y mejorar así la eficiencia global del sistema de transporte público. Sin embargo, según definieron ya Terzis and Last (2000), un intercambiador de transportes para que sea eficiente debe ser no sólo competitivo desde una perspectiva funcional, sino resultar atractivo para los usuarios dado que sus experiencias físicas y sus reacciones psicológicas se ven significativamente influenciadas por su diseño y operación. Además, la calidad del servicio prestado en ellos afecta directamente sobre la experiencia diaria de los viajeros. Sin embargo, todavía no existen normativas a nivel europeo que especifiquen como deberían ser los intercambiadores urbanos de transporte.

\footnotetext{
${ }^{1}$ Autor: Sara.hernandez@arup.com; Tel.: +34 915239276 c/ Alcalá, 54. Madrid (28014), España.
} 
Este estudio presenta herramientas ${ }^{2}$ de análisis dirigidas a planificadores y gestores de los propios intercambiadores que les permita entender mejor el funcionamiento de los mismos y gestionar los recursos disponibles de una manera más eficiente, identificando las fortalezas y debilidades de estas terminales multimodales. Así mismo, el principal objetivo de este trabajo es presentar el marco metodológico de investigación desarrollado junto con los principales resultados obtenidos en los tres intercambiadores de transporte europeos seleccionados como casos de estudio.

El artículo se estructura en las siguientes secciones. La sección 2 presenta un breve estado del arte sobre las necesidades y experiencia del usuario en terminales multimodales. La sección 3 describe el marco metodológico desarrollado para el análisis y evaluación de intercambiadores urbanos de transporte y la descripción de los casos de estudio donde es implementado. Las características de las herramientas de gestión son identificadas en la sección 4. Los principales resultados obtenidos así como algunas conclusiones y políticas de gestión en materia de intercambiadores urbanos son presentados en la sección 5 y sección 6 , respectivamente.

\section{LA EXPERIENCIA DEL USUARIO EN INTERCAMBIADORES URBANOS}

Como se ha mencionado anteriormente, una terminal multimodal actúa de nodo dentro de la red y juega un papel fundamental en el sistema de transporte por el impacto que tiene sobre el tiempo total de viaje (Sun et al., 2012). En general, los usuarios de transporte público emplean entre un 10-30\% de este tiempo esperando en los puntos de conexión para coger el siguiente modo o servicio de transporte (Litman, 2010).Por tanto, es clave identificar cuáles son las necesidades del usuario y qué aspectos y elementos de un intercambiador urbano de transporte afectan y condicionan su experiencia dentro de ellos y de este modo, reducir su sensación de estrés y minimizar los inconvenientes de la transferencia.

Hasta el momento, los estudios se han centrado generalmente en el análisis de la calidad percibida de los servicios de transporte público (dell'Olio et al., 2011; Cavana et al., 2007; Eboli and Mazzulla, 2007; Lin et al., 2007; Nathanail, 2008; Jen et al., 2010). Sin embargo, esta calidad percibida depende de otros aspectos tales como los estándares de las conexiones entre los diferentes modos de transporte (Guo and Wilson, 2007). Algunos estudios recientes han comenzado a investigar la calidad percibida de las infraestructuras de transporte en respuesta al creciente interés en desarrollar instalaciones de transferencia multimodal eficientes.

En relación a los intercambiadores de transporte urbano, hay que tener en cuenta además que se encuentran localizados en ciudades por lo que juegan un papel clave como 'lugares de encuentro'. Es decir, su función como 'un lugar' y no como una barrera aumenta y la creación de sinergias no relacionadas con el transporte es clave para mejorar la experiencia del usuario (Hernandez and Monzon, 2016). Por otra parte, Desiderio (2004) señaló que en entornos urbanos, las terminales multimodales establecen también relaciones entre los usuarios y el territorio y deberían ser consideradas como un elemento de las estrategias de desarrollo urbano dado su papel como 'puertas de la ciudad'. Así mismo, los intercambiadores son lugares donde el usuario no solo pasa a través de ellos sino que además emplea una parte importante de su tiempo (van Hagen, 2011).

En base a todas estas ideas y conceptos, un intercambiador de transportes urbano no debe ser solo definido bajo una perspectiva funcional. Como ya se ha destacado, son los usuarios

\footnotetext{
${ }^{2}$ Estas herramientas de análisis y gestión son parte de la tesis doctoral: 'Assessment methodology to make urban transport interchanges attractive for users' (Hernandez, 2015).
} 
de transporte público quién finalmente determinan la eficiencia de un intercambiador, y por tanto, deben definirse teniendo en cuenta sus necesidades y expectativas desde un enfoque más psicológico.

Pero, ¿cómo debería ser el funcionamiento de un intercambiador?, ¿Qué aspectos funcionales y psicológicos son fundamentales desde el punto de vista del usuario? ¿Cómo reducir las inconveniencias de la transferencia y mejorar la experiencia del usuario? ¿Cómo hacer más atractivos los intercambiadores de transporte urbano para los usuarios?

\section{MARCO METODOLÓGICO DE INVESTIGACIÓN}

Para dar respuesta a todas estas preguntas es necesario conocer y configurar correctamente el contexto de un intercambiador de transportes, para lo que el proceso de toma de datos resulta crucial. Así mismo, la mejor manera de recoger y comprender las opiniones y necesidades de los usuarios acerca de su experiencia en un intercambiador urbano de transportes es a través de una encuesta de satisfacción. Por tanto, la Figura 1 muestra las cuatros etapas desarrolladas como marco metodológico de investigación:

- Diseño y proceso de implementación de la encuesta de satisfacción a usuarios de intercambiadores urbanos de transporte

- Descripción y análisis cualitativo de los casos de estudio europeos seleccionados

- Definición de los perfiles de usuarios y sus patrones de movilidad

- 'Proceso de evaluación': desarrollo y aplicación de las herramientas de análisis desarrolladas a los intercambiadores seleccionados como casos de estudio

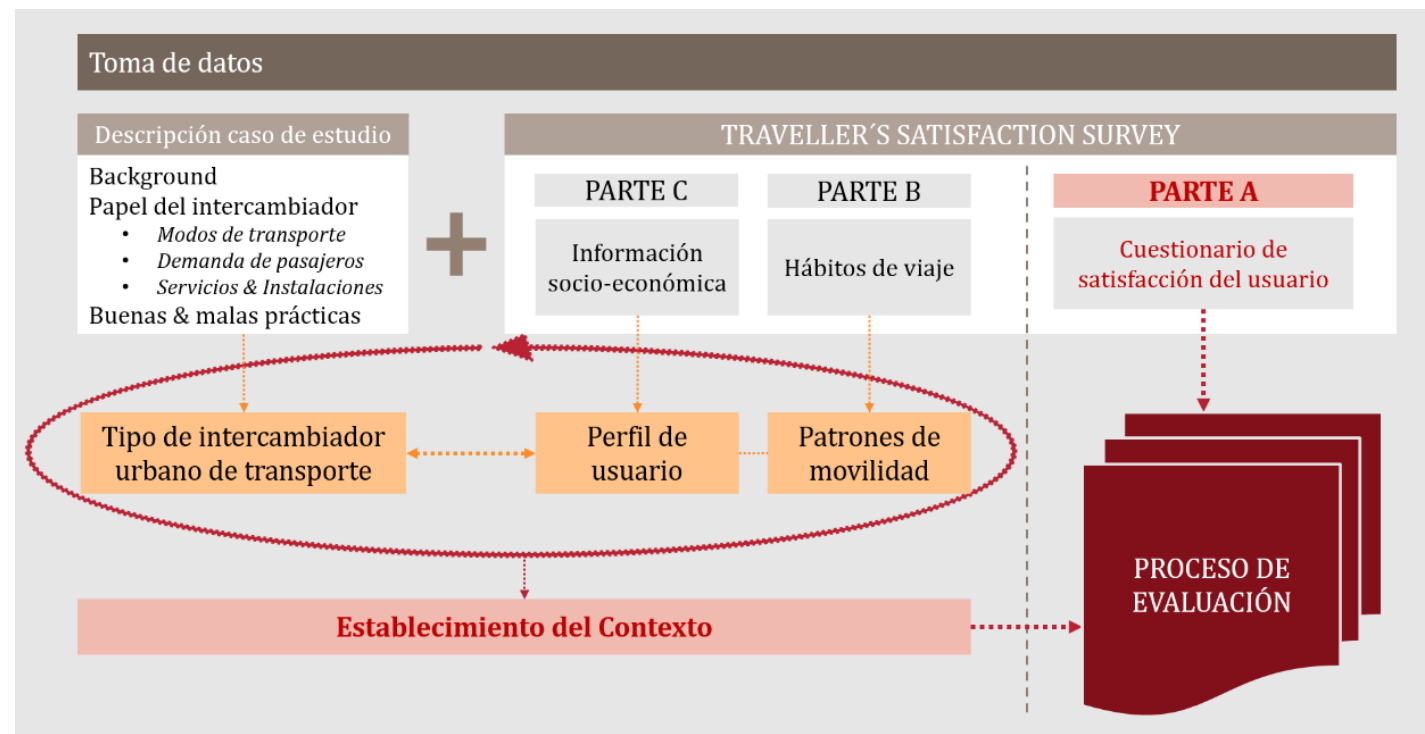

Fig. 1 - Marco metodológico de investigación

\subsection{Encuesta de satisfacción del usuario: diseño e implementación}

Muchos de los aspectos más relevantes que inciden en la toma de decisiones de los usuarios en un viaje multimodal están directamente relacionados con sus características socioeconómicas y sus hábitos de viaje (Yang et al., 2015). Por ello, la encuesta de satisfacción fue diseñada $a d-h o c$ y se estructuró fundamentalmente en tres partes: 
- Parte $\mathrm{A}^{3}$ : cuestionario de satisfacción al usuario

- Parte B: hábitos de viaje

- Parte C: características socioeconómicas

Por otra parte, dado el tipo de movilidad dentro de los intercambiadores urbanos donde los usuarios transfieren rápidamente de un modo o servicio a otro, los métodos tradicionales de entrevistas cara a cara no se consideraron apropiados para llevar a cabo la encuesta diseñada (aproximadamente de una duración de 20 minutos). En consecuencia, se desarrolló un nuevo proceso de implementación que combina las técnicas tradicionales con encuestas on-line ('computer-assisted methods') (ver Figura 2).

En primer lugar, se llevaron a cabo encuestas piloto para comprobar que el cuestionario era comprensible y corregir cualquier tipo de error. En segundo lugar, se realizaron entrevistas breves a los usuarios dentro de los intercambiadores con el fin de explicarles los principales objetivos de la encuesta y se les entregó una tarjeta con el enlace a la página web de la misma. Además, esta tarjeta incluía un número de control para supervisar las encuestas recibidas y evitar la duplicidad de las respuestas e información sobre el sorteo de un iPad 2 del que entraban a formar parte por responder a las preguntas del cuestionario.

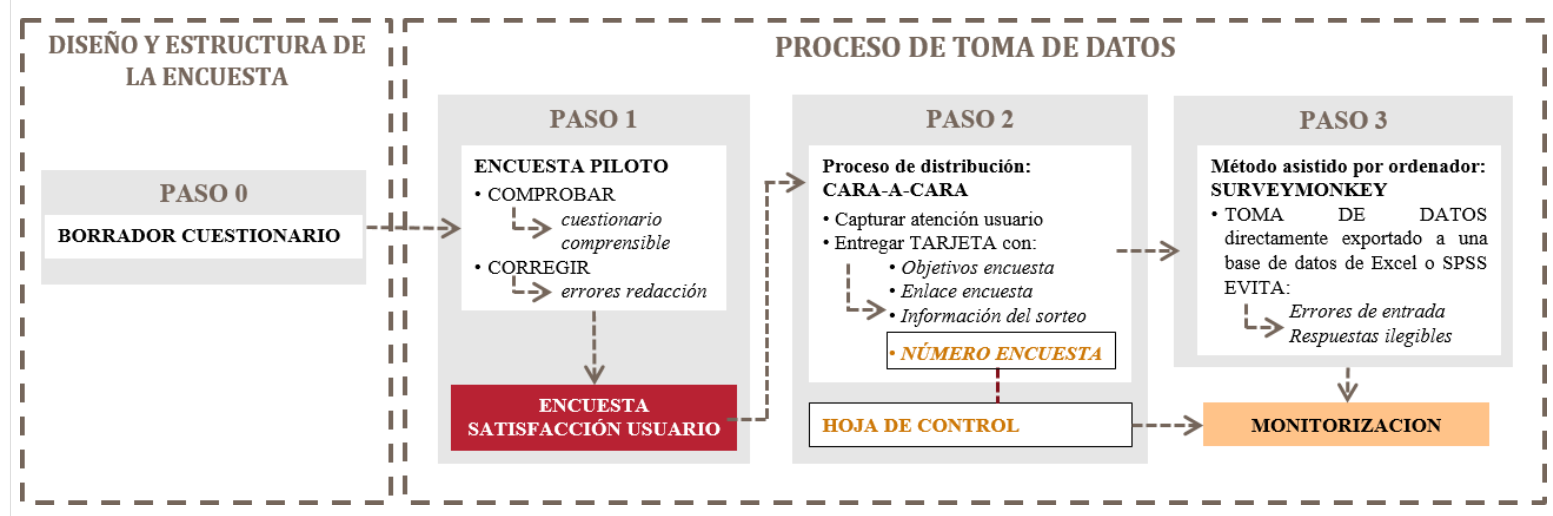

Fig. 2 - Metodología de implementación de encuestas en intercambiadores

Esta encuesta se llevó a cabo gracias a la financiación de la Comisión Europea a través del proyecto City-HUB del $7^{\circ}$ Programa Marco de la UE y tuvo como objetivo proporcionar un mejor entendimiento de las respuestas emocionales del usuario hacia un intercambiador.

\subsection{Descripción de los casos de estudio}

La localización, los modos de transporte y las características de los usuarios son variables clave que determinan la categoría de una terminal de transporte de viajeros multimodal (Pitsiava-Latinopoulou and Iordanopoulos, 2012). Los casos de estudio fueron seleccionados por tanto para proporcionar un balance en términos geográficos, buscando heterogeneidad entre modos de transporte, tamaño del intercambiador y su papel dentro del ámbito urbano. Además, la tipología de terminal fue seleccionada siguiendo los criterios de clasificación desarrollados por PORTAL (2003) de acuerdo a su localización dentro de la red de transportes: intercambiador periférico, sub-céntrico y céntrico, respectivamente. Los casos estudio seleccionados fueron la estación de tren de Ilford (Londres, Reino Unido), el

\footnotetext{
${ }^{3}$ El cuestionario de satisfacción ('Parte $A$ ') consta de 37 variables observadas relacionadas con distintos aspectos y elementos de un intercambiador así como una pregunta relacionada con la valoración global. El usuario responde a cada aspecto en una escala Likert de 1 (completamente insatisfecho) a 5 (completamente satisfecho)
} 
intercambiador de transportes de Moncloa (Madrid, España) y el intercambiador de transportes de Kamppi (Helsinki, Finlandia).

La Tabla 1 proporciona una breve descripción de las características principales de cada uno de ellos así como la demanda de pasajeros diaria de los principales modos de transporte. Kamppi y Moncloa son intercambiadores urbanos prácticamente nuevos, construidos y reformados en 2006 y 2008, respectivamente. Por el contrario, Ilford fue construida en 1839 y reformado posteriormente en 1980. Los usuarios de Moncloa y Kamppi utilizan fundamentalmente el metro y el autobús, mientras que los usuarios de Ilford viajan en tren. Los servicios de transporte de Moncloa son básicamente de ámbito local y regional mientras que el intercambiador de Kamppi alberga a su vez los principales servicios de autobús de ámbito nacional y una línea de servicio internacional (St. Petersburg). En cuanto a Ilford, sus servicios de transporte son de ámbito local.

\begin{tabular}{|c|c|c|c|c|c|}
\hline $\begin{array}{l}\text { Intercambiador } \\
\text { urbano de } \\
\text { transportes }\end{array}$ & $\begin{array}{l}\text { Año } \\
\text { construcción }\end{array}$ & Modos de transporte (1) & $\begin{array}{r}\text { Pasajeros/día } \\
\text { (2013) }\end{array}$ & Ámbito & Descripción \\
\hline $\begin{array}{l}\text { Estación de tren } \\
\text { de Ilford }\end{array}$ & $\begin{array}{l}1839 \\
\text { Reconstruido } \\
\text { en } 1980 \\
\text { (Planificado } \\
\text { para reforma) }\end{array}$ & $\begin{array}{l}\text { Tren } \\
\text { Autobús } \\
\text { Bicicleta (parking) } \\
\text { Vehículo privado } \\
\text { (estacionamiento para } \\
\text { recoger/dejar pasajeros) } \\
\text { Parking vehículo privado } \\
\text { Taxi }\end{array}$ & 22,405 & $\begin{array}{l}\text { Local + } \\
\text { Regional }\end{array}$ & $\begin{array}{l}\text { Núcleo suburbano en el } \\
\text { distrito londinense de } \\
\text { Redbridge. Estación } \\
\text { localizada en el centro. } \\
\text { Centro comercial enfrente } \\
\text { de la estación. } \\
\text { (Intercambiador } \\
\text { periférico) }\end{array}$ \\
\hline Moncloa & $\begin{array}{l}1995 \\
\text { Reformado en } \\
2008\end{array}$ & $\begin{array}{l}56 \text { líneas bus } \\
\text { metropolitanos } \\
3 \text { líneas bus urbano } \\
2 \text { líneas de metro } \\
2 \text { líneas bus larga distancia } \\
\text { No parking vehículo privado }\end{array}$ & $\begin{array}{r}59,989 \\
96,789 \\
109,321\end{array}$ & $\begin{array}{l}\text { Local + } \\
\text { Regional + } \\
\text { Nacional } \\
\text { (2 líneas) }\end{array}$ & $\begin{array}{l}\text { Puerta de entrada a } \\
\text { Madrid. Área con } \\
\text { monumentos históricos } \\
\text { cerca del centro de la } \\
\text { ciudad. Línea } 6 \text { de metro } \\
\text { que conecta con todos los } \\
\text { puntos centrales de Madrid. } \\
\text { (Intercambiador sub- } \\
\text { céntrico) }\end{array}$ \\
\hline Kamppi & $\begin{array}{l}2005 \\
\text { (Inicio de } \\
\text { operación) } \\
2006 \\
\text { (Centro } \\
\text { comercial) }\end{array}$ & $\begin{array}{l}21 \text { líneas bus urbano } \\
40 \text { líneas bus regional } \\
\mathbf{1 5} \text { líneas bus } \\
\text { metropolitanos } \\
\mathbf{1} \text { línea de metro } \\
1 \text { línea bus internacional } \\
2 \text { líneas tranvía (red urbana) } \\
\text { Centro bicicletas (parking, } \\
\text { alquiler, servicio) } \\
\text { Parking vehículo privado } \\
\text { Taxis }\end{array}$ & $\begin{array}{r}19,360 \\
8,500 \\
7,500 \\
21,700\end{array}$ & $\begin{array}{l}\text { Local + } \\
\text { Regional + } \\
\text { Nacional + } \\
\text { Internacional } \\
\text { (1 bus a St. } \\
\text { Petersburg) }\end{array}$ & $\begin{array}{l}\text { Intercambiador ubicado en } \\
\text { el centro de Helsinki. } \\
\text { Centro comercial. } \\
\text { Estación de tren a } 500 \mathrm{~m} \\
\text { del intercambiador de } \\
\text { Kamppi. } \\
\text { (Intercambiador céntrico) }\end{array}$ \\
\hline
\end{tabular}

(1) Se indican en negrita los modos de transporte más relevantes de cada intercambiador y su demanda

\section{Tabla 1 - Principales características de los casos de estudio}

Cabe destacar que los tres intercambiadores juegan un papel clave en los viajes multimodales de sus correspondientes ciudades cubriendo un amplio espectro de tipologías de intercambiador y dispersión geográfica.

\section{HERRAMIENTAS DE GESTIÓN PARA EL ANÁLISIS DE INTERCAMBIADORES URBANOS}

Esta sección presenta la cuarta y última etapa del marco metodológico de investigación: el 'Proceso de evaluación' (Figura 3). Este marco metodológico permite a los gestores de los intercambiadores formular decisiones estratégicas adecuadas orientadas, no sólo a reducir las inconveniencias de la transferencia, sino también a mejorar las percepciones del usuario 
sobre la calidad del servicio.

El 'Proceso de evaluación', basado en la experiencia del usuario, trata de dar respuesta a cómo es el rendimiento de un intercambiador de transportes existente y como debería ser, preguntas clave orientadas a la gestión y planificación de intercambiadores urbanos. El proceso consta de tres herramientas que tiene por objetivo proporcionar un conocimiento profundo del funcionamiento del intercambiador con el fin de entender y gestionar adecuadamente los recursos disponibles, respectivamente. El último paso es definir directrices generales de planificación de intercambiadores eficientes bajo un doble enfoque: aspectos funcionales y psicológicos.

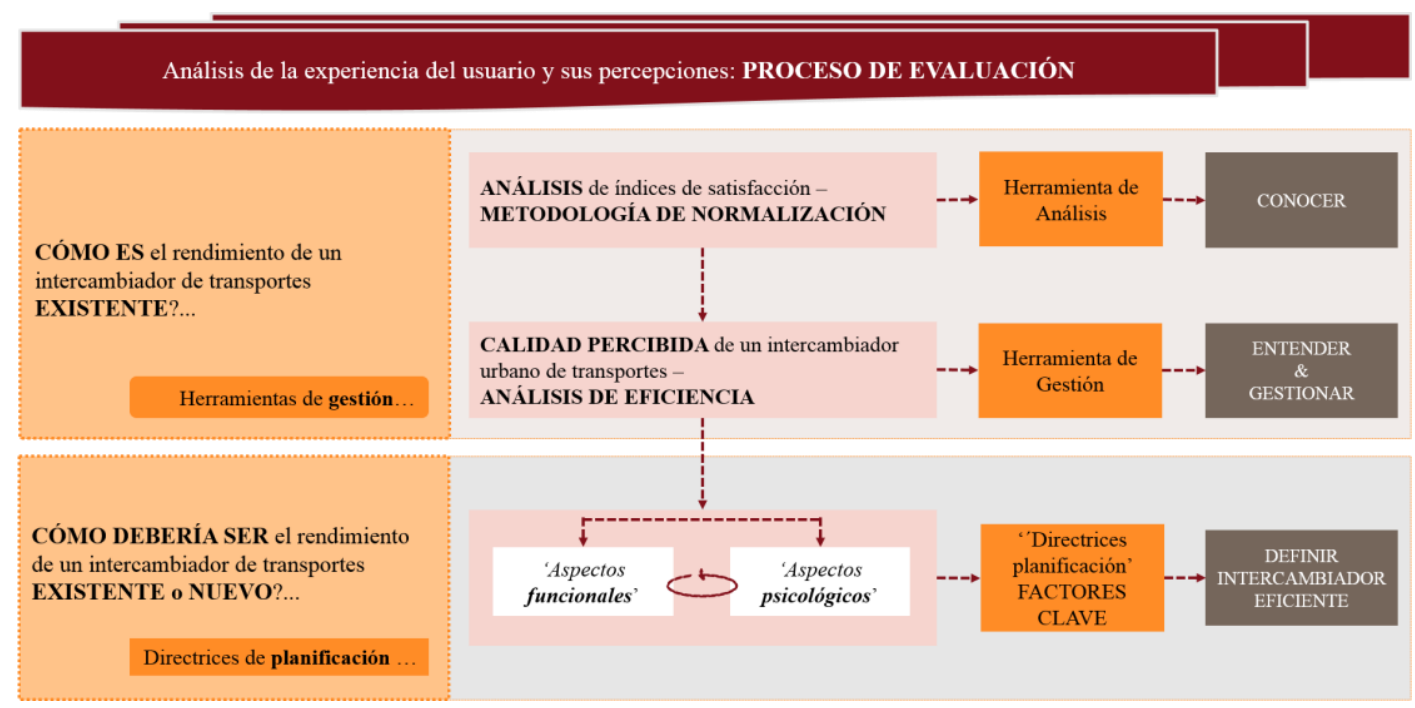

Fig. 3 - Proceso evaluación para el análisis y gestión de intercambiadores urbanos

\subsection{Análisis de eficiencia de un intercambiador de transportes}

En primer lugar se lleva cabo un análisis de los índices de satisfacción de los usuarios y se identifican, independientemente del contexto social y cultural de cada intercambiador, los aspectos y elementos controvertidos de su diseño y operación (herramienta de análisis metodología de normalización).

En segundo lugar, la herramienta de gestión calcula la 'importancia derivada' de cada elemento o variable observada proporcionando un carácter bidimensional al análisis. Esta herramienta a su vez permite identificar las fortalezas y debilidades de cada intercambiador de transportes. Esta herramienta, desarrollada por (Hernandez et al., 2016), es un procedimiento analítico que combina 2 metodologías:

- Modelo CART (Classification and Regression Trees)

- Objetivo $\rightarrow$ obtener la importancia derivada de cada variable observada

- Análisis IPA (Importance-Performance Analysis)

- Objetivo $\rightarrow$ identificar qué aspectos o elementos de un intercambiador necesitan mejorar y cuál debe de ser su prioridad.

\subsection{Factores clave en el diseño y operación de un intercambiador de transportes}

Por último, el tercer paso del 'Proceso de evaluación' es identificar los factores clave de diseño y operación de un intercambiador urbano con el objetivo de definir directrices 
generales de planificación. La metodología aplicada se basa en un Análisis de Componentes Principales que cubre el conjunto de las 37 variables observadas. Este análisis reduce el conjunto de datos a un tamaño más manejable con el fin de comprender e interpretar la estructura del conjunto de variables (Field, 2009). Dicho análisis, aplicado a los tres casos de estudio seleccionados (sección 3.2), fue llevado a cabo por Hernandez and Monzon (2016).

\section{RESULTADOS}

Las encuestas se llevaron a cabo en los tres casos de estudio seleccionados entre mayo y octubre de 2013. Fueron recibidas 1.471 encuestas $\left(\mathrm{N}_{\text {Ilford }}=234 ; \mathrm{N}_{\text {Moncloa }}=928\right.$ y $\mathrm{N}_{\text {Kamppi }}=$ 309) y tras descartar las encuestas incompletas o mal respondidas, hubo una muestra total de 1.389 encuestas válidas ( $\mathrm{N}_{\text {Ilford }}=226 ; \mathrm{N}_{\text {Moncloa }}=865$ y $\left.\mathrm{N}_{\text {Kamppi }}=298\right)$.

La calidad tanto de las infraestructuras como de los servicios percibida por los usuarios de intercambiadores urbanos normalmente depende del contexto del intercambiador (Hine and Scott, 2000; Redman et al., 2013; Abreu e Silva and Bazrafshan, 2013; Harmer et al., 2014). Algunas de las cuestiones que pueden influir directamente en las percepciones de los usuarios son los principales modos de transporte involucrados, la ubicación del intercambiador, la antigüedad de la infraestructura y los propios perfiles de usuario y sus patrones de movilidad. Sin embargo, el análisis de los resultados muestra que independientemente del contexto de cada intercambiador, existen 8 factores de diseño y operación comunes en todos ellos: 'Información', 'Condiciones de transferencia', 'Seguridad', 'Situaciones de emergencia', 'Diseño e imagen', 'Calidad ambiental', 'Servicios e instalaciones' y 'Confort durante el tiempo de espera'. Estos factores definen un intercambiador eficiente desde dos perspectivas diferentes que confirman los supuestos teóricos y conceptuales previos basados en la revisión de la literatura: perspectiva funcional y perspectiva psicológica. Como consecuencia de ello, un intercambiador debe ser concebido tanto como 'un nodo de transporte' como 'un lugar' (ver Figura 4).

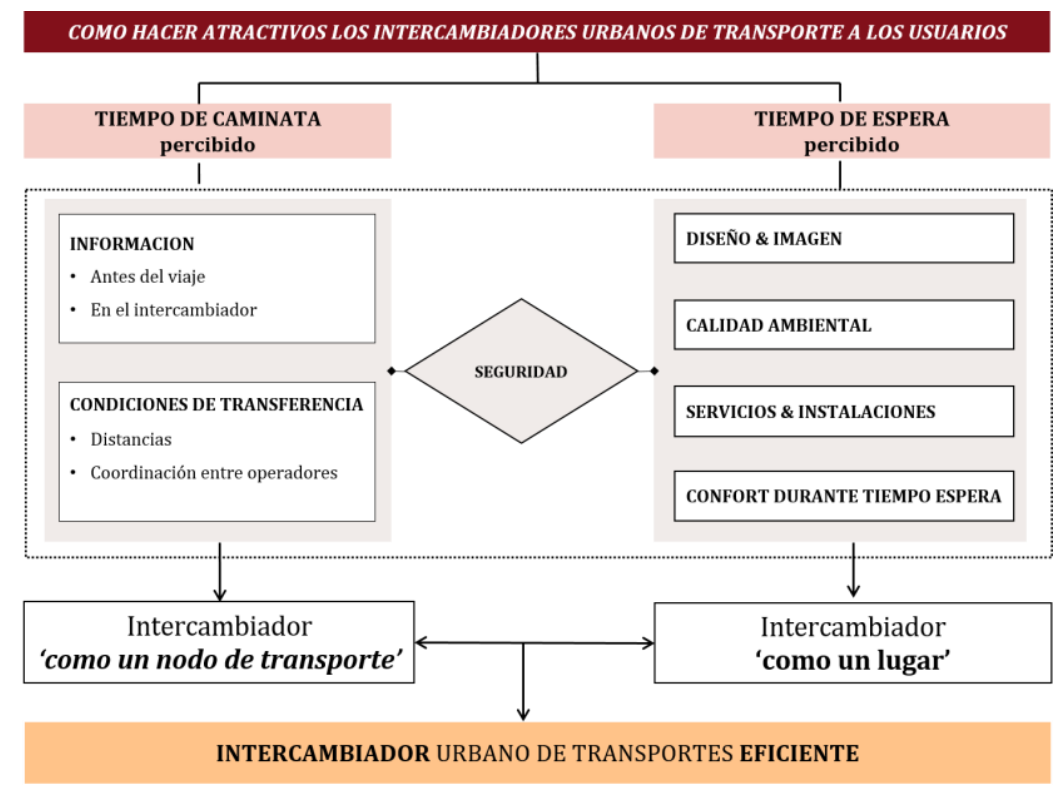

\section{Fig. 4 - Factores clave de diseño y operación de un intercambiador urbano}

Por otro lado y de acuerdo a la tipología específica de cada intercambiador, se han identificado algunos resultados particulares:

- La estación de Ilford es fundamentalmente una estación de ferrocarril donde el perfil del usuario es un usuario habitual que viaja solo (94.2\%) y principalmente por 
motivos de trabajo (81.4\%). El tiempo total de viaje es de 37.3 min y su tiempo de espera en el intercambiador de $7.4 \mathrm{~min}$, lo que supone aproximadamente un $20 \%$ de su tiempo total de viaje.

Los aspectos identificados como más importantes están directamente relacionados con la actividad del transporte: 'Provisión de información' y 'Seguridad'. Por lo tanto, los usuarios perciben el intercambiador como 'un nodo de transporte'. Así mismo, las principales debilidades del intercambiador son el 'diseño interno' y 'la sensación de seguridad en áreas de transferencia y espera en horas de nocturnidad'

- Por el contrario, el intercambiador de Kamppi se caracteriza por su actividad comercial dada su ubicación en la planta baja de un nuevo centro comercial en el centro de la ciudad de Helsinki (Finlandia). Esto explica por qué la mayoría de los aspectos identificados como más importantes están relacionados con la 'Imagen y el atractivo' y la 'Comodidad y confort'. En este caso, el usuario percibe el intercambiador más 'como un lugar' en sí mismo. Por otro lado, las principales debilidades son la 'coordinación entre operadores' y la facilidad de 'movimiento' debido a la aglomeración dentro del intercambiador. Señalar que actualmente el intercambiador está operando cerca de sus máximos de capacidad.

En cuanto al perfil del usuario, el $62 \%$ es usuario habitual que fundamentalmente viaja solo (90.3\%) por motivos de trabajo (55.4\%). Sin embargo, el otro $38 \%$ se identifica con un usuario no habitual que viaja por motivos de ocio (30.5\%). El tiempo total de viaje es de 55.5 min y el tiempo de espera representa el $25 \%$ de este tiempo (13.8 $\mathrm{min})$.

- Sin embargo, el caso de estudio del intercambiador de Moncloa representa un balance entre ambos enfoques. Los aspectos identificados como más importantes por los usuarios son por un lado la 'Seguridad' y 'Provisión de información', particularmente la señalización dentro del intercambiador. Estos aspectos definen un intercambiador desde su perspectiva funcional de 'nodo de transporte'. Y por otro lado, se identifica como igualmente importante el 'Confort y la comodidad', señalando el intercambiador 'como un lugar'. Como principales debilidades destacan la calidad de la 'información proporcionada' y la 'capacidad de asiento'. En este sentido señalar ambas debilidades están directamente relacionadas con el contexto del intercambiador ya que no existe actualmente información en tiempo real y en segundo lugar existe un claro problema de gestión de colas durante el tiempo de espera.

En vista a los resultados obtenidos en los tres casos de estudio, cabe destacar su directa relación con el contexto de cada intercambiador y por lo tanto, ponen de manifiesto la efectividad del 'Proceso de evaluación' desarrollado.

\section{CONCLUSIONES}

Muchos usuarios de transporte público hoy en día necesitan combinar varios modos o servicios de transporte para llegar a su destino final, y los inconvenientes causados por estas transferencias ha dado lugar a una disminución de la capacidad de atracción del transporte público (Guo and Wilson, 2011). Los intercambiadores urbanos de transporte son por tanto una experiencia diaria de estos usuarios y se han convertido en elementos fundamentales para mejorar su experiencia durante la ruptura del viaje. Sin embargo, existen muy pocos métodos de evaluación que midan las necesidades de los usuarios con respecto a estas infraestructuras.

Este estudio presenta tres herramientas de análisis dirigidas a planificadores y gestores de los propios intercambiadores que permiten entender mejor el funcionamiento de los mismos 
y gestionar de una manera más eficiente los recursos disponibles, identificando a su vez las fortalezas y debilidades de estas terminales multimodales.

En particular, condiciones relacionadas con la seguridad son aspectos psicológicos indispensables para los usuarios y están directamente relacionados con el rendimiento general del intercambiador. Además, a pesar de las diferencias significativas en las características y contexto de cada intercambiador, los factores de diseño y operación identificados son comunes en todos ellos. En base a estos resultados, para que un intercambiador urbano de transportes sea eficiente debe cumplir cuatro principios básicos en línea a su doble naturaleza:

\section{'Como nodo de transportes'}

- Reducir el tiempo de espera. Son cruciales las distancias de transferencia y la coordinación entre operadores

- Hacer más fácil el uso del intercambiador y reducir los niveles de estrés de los usuarios. La información de viaje y la señalización son los aspectos más importantes.

\section{'Como lugar'}

- Hacer la estancia más confortable. Aspectos como el diseño interno y externo, la calidad del aire y la temperatura y pantallas de información son elementos fundamentales

- Mejorar el uso del tiempo en el intercambiador. Los servicios e instalaciones y la disponibilidad de señal telefónica y WiFi pueden contribuir a lograr dicho propósito

En conclusión, los intercambiadores urbanos pueden albergar otro tipo de actividades y actuar no sólo como nodos de transporte, sino también como lugares de encuentro dentro de las ciudades. Sin embargo, su diseño debe estar centrado en su perspectiva funcional como elemento de la red de transportes, de lo contrario las instalaciones no serían más que un centro comercial con buenas conexiones de transporte. Por último, como ya señaló Scott (2003), estas terminales multimodales pueden ser consideradas como elementos fundamentales en el desarrollo futuro de las ciudades.

\section{REFERENCIAS}

Abreu e Silva, J., Bazrafshan, H., 2013. User Satisfaction of Intermodal Transfer Facilities in Lisbon, Portugal: Analysis with Structural Equations Modeling. Transp. Res. Rec. J. Transp. Res. Board 2350, 102-110. doi:10.3141/2350-12

Banister, D., 2011. The trilogy of distance, speed and time. J. Transp. Geogr. 19, 950-959. doi:10.1016/j.jtrangeo.2010.12.004

Cavana, R.., Corbett, L.., Lo, Y.., 2007. Developing zones of tolerance for managing passenger rail service quality. Int. J. Qual. Reliab. Manag. 24, 7-31. doi:10.1108/02656710710720303

COM, 2006. COMMUNICATION FROM THE COMMISSION TO THE COUNCIL AND THE EUROPEAN PARLIAMENT. Keep Europe moving - Sustainable mobility for our continent Mid-term review of the European Commission's 2001 Transport White Paper. Office for Official Publications of the European Communities, Luxembourg.

dell'Olio, L., Ibeas, A., Cecin, P., 2011. The quality of service desired by public transport users. Transp. Policy 18, 217-227. doi:10.1016/j.tranpol.2010.08.005

Desiderio, N., 2004. Requirements of Users and Operators on the Design and Operation of Intermodal Interchanges. Technishe Universitat Darmstadt, Fachgebiet Verkehrsplanung und Verkehrstechnik.

Eboli, L., Mazzulla, G., 2007. Service quality attributes affecting customer satisfaction for bus transit. World Transit Res.

Field, A.P., 2009. Discovering Statistics Using SPSS: (and Sex, Drugs and Rock'n'roll), Third Edition. ed. SAGE. 
Guo, Z., Wilson, N., 2007. Modeling the Effects of Transit System Transfers on Travel Behavior: Case of Commuter Rail and Subway in Downtown Boston, Massachusetts. Transp. Res. Rec. J. Transp. Res. Board 2006, 11-20.

Guo, Z., Wilson, N.H.M., 2011. Assessing the cost of transfer inconvenience in public transport systems: A case study of the London Underground. Transp. Res. Part Policy Pract. 45, $91-$ 104. doi:10.1016/j.tra.2010.11.002

Harmer, C., Millard, K., Palmer, D., Ubbels, B., Monzon, A., Hernández, S., 2014. What makes a successful urban interchange?: results from an evidence review. Presented at the Transport Research Arena (TRA) 5th Conference: Transport Solutions from Research to Deployment.

Heilig, G.K., 2012. World Urbanization Prospects, the 2011 Revision (Report). United Nations, Department of Economic and Social Affairs (DESA), Population Division, Population Estimates and Projections Section, New York.

Hernandez, S., 2015. Assessment methodology to make urban transport interchanges attractive for users (Ph.D. Thesis). Universidad Politecnica de Madrid, Spain.

Hernandez, S., Monzon, A., 2016. Key factors for defining an efficient urban transport interchange: Users' perceptions. Cities 50, 158-167. doi:10.1016/j.cities.2015.09.009

Hernandez, S., Monzon, A., de Oña, R., 2016. Urban transport interchanges: A methodology for evaluating perceived quality. Transp. Res. Part Policy Pract., Practical Applications of Novel Methodologies to Real Cases: Selected Papers from the XIII Pan-American Conference on Traffic and Transportation Engineering 84, 31-43. doi:10.1016/j.tra.2015.08.008

Hine, J., Scott, J., 2000. Seamless, accessible travel: users' views of the public transport journey and interchange. Transp. Policy 7, 217-226. doi:10.1016/S0967-070X(00)00022-6

Jen, W., Tu, R., Lu, T., 2010. Managing passenger behavioral intention: an integrated framework for service quality, satisfaction, perceived value, and switching barriers. Transportation 38, 321342. doi:10.1007/s11116-010-9306-9

Lin, J.-H., Lee, T.-R., Jen, W., 2007. Assessing asymmetric response effect of behavioral intention to service quality in an integrated psychological decision-making process model of intercity bus passengers: a case of Taiwan. Transportation 35, 129-144. doi:10.1007/s11116-0079139-3

Litman, T., 2010. Transportation Cost and Benefit Analysis II-Travel Time Costs Victoria.

Lopez-Lambas, M.E., Monzon, A., 2010. Private funding and management for public interchanges in Madrid. Res. Transp. Econ., Reforming Public Transport throughout the World 29, 323328. doi:10.1016/j.retrec.2010.07.041

Nathanail, E., 2008. Measuring the quality of service for passengers on the hellenic railways. Transp. Res. Part Policy Pract. 42, 48-66. doi:10.1016/j.tra.2007.06.006

Neirotti, P., De Marco, A., Cagliano, A.C., Mangano, G., Scorrano, F., 2014. Current trends in Smart City initiatives: Some stylised facts. Cities 38, 25-36. doi:10.1016/j.cities.2013.12.010

Pitsiava-Latinopoulou, M., Iordanopoulos, P., 2012. Intermodal Passengers Terminals: Design Standards for Better Level of Service. Procedia - Soc. Behav. Sci., Transport Research Arena 2012 48, 3297-3306. doi:10.1016/j.sbspro.2012.06.1295

PORTAL, 2003. Integrated transport chains. Transport Teaching Material. European Comission and Research for Sustainable Mobility.

Redman, L., Friman, M., Gärling, T., Hartig, T., 2013. Quality attributes of public transport that attract car users: A research review. Transp. Policy 25, 119-127. doi:10.1016/j.tranpol.2012.11.005

Scott, F., 2003. InterchangeABLE. Helen Hamlyn Centre, Royal College of Art, London.

Sun, L., Rong, J., Yao, L., Xu, H., Liu, H., 2012. Entropy-based estimation of transfers in a terminal. Transp. Plan. Technol. 35, 303-315. doi:10.1080/03081060.2012.671038

Terzis, G., Last, A., 2000. GUIDE - "Urban Interchanges - A Good Practice Guide" (Final Report), European 4th RTD Framework Programme.

van Hagen, M., 2011. Waiting experience at train stations (Ph.D. Thesis). Twenty University, The Netherlands.

Yang, M., Zhao, J., Wang, W., Liu, Z., Li, Z., 2015. Metro commuters' satisfaction in multi-type access and egress transferring groups. Transp. Res. Part Transp. Environ. 34, 179-194. doi:10.1016/j.trd.2014.11.004 